\title{
Estudos preliminares de microrganismos eficientes (EM) na biorremediação da água da barragem da vila Bananeira em Arapiraca-Alagoas, Brasil
}

\author{
Preliminary studies of the action of eficiente microorganisms (EM) in the biorremediation of water
}

from the Bananeira village dam in Arapiraca -Alagoas, Brazil

Estudios preliminares de microorganismos eficientes (EM) en la biorremediación del agua de la represa de Vila Bananeira en Arapiraca-Alagoas, Brasil

\section{Resumo}

O seguinte trabalho teve como objetivo analisar o efeito de inóculos de Microrganismos Eficientes (EM) na biorremediação de água contaminada por dejetos animais, como também, os parâmetros físico-químicos e microbiológicos do fermentado de EM e de água contaminada. O EM foi capturado e ativado através do método caseiro. O protocolo para coleta das amostras da água contaminada e EM foi de acordo com Standard Methods for the Examination of water and Waste Water. Foram investigados os coliformes totais e termotolerantes tanto da água quanto do EM, através da técnica do número mais provável (NMP), conhecida como o método de tubos múltiplos. Foram avaliados os parâmetros físico-químicos $(\mathrm{pH}$, sólidos totais, sódio, nitrito, nitrato, cloretos, dureza total, cor aparente, condutividade e turbidez) da água contaminada e do EM. Para avaliar o potencial biorremediador do EM, foram realizados 10 testes com diferentes porcentagens de diluição dos microrganismos, que seguiram as seguintes concentrações: 0,$3 ; 1,0 ; 3,0 ; 20 ; 25 ; 30 ; 35 ; 40 ; 45$ e 50 \% de EM. Foi possível constatar que os parâmetros físicoquímicos analisados atendem aos pré-requisitos do Ministério da Saúde no que tange a qualidade da água, no entanto, quanto aos parâmetros microbiológicos, $99 \%$ das amostras mostraram-se impróprias para o consumo humano. O fermentado de microrganismos eficientes se encontra livre do grupo de coliformes. Quanto a biorremediação utilizando EM, as baixas concentrações de 0,3 a 3 não remediaram o grupo coliformes, no entanto, verificou-se que as concentrações graduais de no mínimo $25 \%$ de EM podem remediar até $78 \%$ do grupo coliformes.

Palavras-chave: Coliformes totais; Coliformes termotolerantes; Parâmetros físico-químicos; Poluentes ambientais.

\begin{abstract}
The following work aimed to analyze the effect of Efficient Microorganisms (EM) on the bioremediation of water contaminated by animal waste as well as the physical-chemical and microbiological parameters of the fermented EM and contaminated water. The EM was captured and activated using the homemade method. The protocol for collecting contaminated water and EM samples was in accordance with Standard Methods for the Examination of Water and Wastewater. Total and thermotolerant coliforms of both water and EM were investigated, using the most
\end{abstract}


probable number (MPN) technique, known as the multiple tube method. The physical-chemical parameters (pH, total solids, sodium, nitrite, nitrate, chlorides, total hardness, apparent color, conductivity, and turbidity) of the contaminated water and the EM were evaluated. To assess the bioremediation potential of EM, 10 tests were performed with different percentages of dilution of microorganisms, which followed the following concentrations: $0.3 ; 1.0 ; 3.0 ; 20 ; 25 ; 30 ; 35 ; 40 ; 45$ and $50 \%$ of EM. It was possible to verify that the physical-chemical parameters analyzed meet the prerequisites of the Ministry of Health with regard to water quality, however, regarding microbiological parameters, $99 \%$ of the samples proved to be unfit for human consumption. The fermentation of efficient microorganisms is free of the coliform group. As for bioremediation using EM low concentrations of 0.3 to 3 did not remedy the coliform group, however it was found that gradual concentrations of at least $25 \%$ of EM can remedy up to $78 \%$ of the coliform group

Keywords: Total coliforms; Thermotolerant coliforms; Physical-chemical parameters; Environmental pollutants.

\section{Resumen}

El siguiente trabajo tuvo como objetivo analizar el efecto del inóculo de Microorganismos Eficientes (EM) en la biorremediación de aguas contaminadas por desechos animales, así como los parámetros fisicoquímicos y microbiológicos del agua EM fermentada y contaminada. EM fue capturado y activado utilizando el método casero. El protocolo para recolectar agua contaminada y muestras de EM se realizó de acuerdo con los Métodos Estándar para el Examen de Agua y Aguas Residuales. Los coliformes totales y termotolerantes tanto en agua como en EM se investigaron utilizando la técnica del número más probable (NMP), conocida como método de tubos múltiples. Se evaluaron los parámetros fisicoquímicos $(\mathrm{pH}$, sólidos totales, sodio, nitrito, nitrato, cloruros, dureza total, color aparente, conductividad y turbidez) del agua contaminada y EM. Para evaluar el potencial de biorremediación de EM, se realizaron 10 pruebas con diferentes porcentajes de dilución de microorganismos, que siguieron las siguientes concentraciones: 0,$3 ; 1,0 ; 3,0 ; 20 ; 25 ; 30 ; 35 ; 40 ; 45$ y 50\% EM. Se pudo verificar que los parámetros físico-químicos analizados cumplen con los prerrequisitos del Ministerio de Salud en cuanto a la calidad del agua, sin embargo, en cuanto a los parámetros microbiológicos, el $99 \%$ de las muestras resultaron no aptas para el consumo humano. El fermentado de microorganismos eficientes está libre del grupo de coliformes. En cuanto a la biorremediación usando EM, bajas concentraciones de 0.3 a 3 no remediaron el grupo de coliformes, sin embargo se encontró que concentraciones graduales de al menos $25 \%$ de EM pueden remediar hasta el $78 \%$ del grupo de coliformes.

Palabras clave: Coliformes totales; Coliformes termotolerantes; Parámetros fisicoquímicos; Contaminantes ambientales.

\section{Introdução}

Dentre os recursos naturais, a água é um dos mais importantes do planeta e seu uso vem se intensificando nas últimas décadas com o desenvolvimento econômico e o aumento populacional, tanto no que se refere ao aumento da quantidade demandada para determinada utilização, quanto à variedade de sua utilização (Silva et al., 2014).

Segundo Santos et al. (2010), manter os padrões de quantidade e qualidade da água representa um desafio à sociedade, já que a maior parte dos problemas relacionados aos recursos hídricos têm como principais problemas a falta de planejamento e gestão, além da sua contaminação. Este novo olhar vem desencadeando ações e estratégias de preservação do meio ambiente, de ocupação do solo e de desenvolvimento de processos produtivos menos danosos (Moraes et al. 2014).

Entre as biotecnologias utilizadas para a recuperação de áreas contaminadas, os microrganismos eficientes (EM) consistem em uma combinação de microrganismos completamente naturais (lactobacilos, leveduras, bactérias fototrópicas e actinobactérias) que, quando coexistem, apresentam um efeito sinérgico que é maior que a soma de seus membros individuais, podendo, entre tantas aplicações, solucionar problemas ambientais como, por exemplo, tratar águas residuais. O uso de microrganismos como ferramentas para a remediação de ambientes contaminados é chamado de biorremediação. De acordo Cotta et al. (2020) a biorremediação também é uma alternativa atraente para descontaminação de solos, demonstrando assim sua ampla utilização. Este processo pode ser realizado por meio de um ou mais consórcios microbianos, indígenas ou não, para a degradação de contaminantes orgânicos poluentes (Pereira et al., 2003). Segundo Cuba et al. (2019) este é um cenário de sucesso para descontaminação de águas, visto que podem apresentar custos econômicos baixos, estando diretamente relacionado aos processos biológicos de microrganismos não patogênicos, ou seja, sem degradação de poluentes.

De acordo com Tavares (2013), essa é uma tecnologia que apresenta, atualmente, um crescimento rápido, sobretudo 
em colaboração com a engenharia genética, utilizada para desenvolver linhagens de microrganismos que tenham capacidade de lidar com poluentes específicos. Sob condições adequadas, os microrganismos ajudam ou são responsáveis por reações químicas que resultam em compostos que apresentam baixo ou nenhum risco aos seres vivos (Moreira \& Dourado, 2007).

Nesse contexto, o presente trabalho teve como principal objetivo avaliar o efeito dos microrganismos eficientes (EM) na biorremediação de águas contaminadas por dejetos animais da barragem da Vila Bananeira, em Arapiraca - AL.

\section{Metodologia}

As amostras de água para avaliação da biorremediação foram coletadas na Barragem da Vila Bananeiras (950'33.6"S 36³4'15.8”O) situado na zona rural do município de Arapiraca-AL, Brasil. A Coleta do EM foi realizada em uma Reserva Particular do Patrimônio Natural em Junqueiro - RPPN Madeiras (9 $51^{\prime} 53.5^{\prime \prime S} 36^{\circ} 20^{\prime} 00.2^{\prime \prime O}$ ) e sua ativação foi no Laboratório de Recursos Florestais, enquanto as análises microbiológicas foram realizadas no Laboratório de Análises Microbiológicas, ambos do Polo Tecnológico Agroalimentar de Arapiraca, da Universidade Estadual de Alagoas. Já as análises físico-químicas, foram encaminhadas para o laboratório Central Analítica. O EM foi preparado e ativado através do método caseiro de captura e preparo do solo (Siqueira et al. 2013). Para captura, foi utilizado arroz cozido sem sal e colocado em bandejas cobertas por uma fina tela de proteção. As bandejas foram deixadas em mata virgem, coberta com serapilheira por aproximadamente 15 dias. Para ativação, foi utilizado melaço e água sem cloro, a mistura foi colocada em recipiente com tampa onde ficou fermentando por 15 dias.

O procedimento de coleta seguiu o protocolo conforme Standard Methods for The Examination of Water and Wastewater (Hunt \& Rice, 2005). As coletas foram em julho de 2019, utilizando recipiente de inox e, para armazenar a água, frasco de polipropileno, todos previamente autoclavados á $121^{\circ} \mathrm{C} \mathrm{em} 1 \mathrm{kpa}$ por $60 \mathrm{~min}$, logo após foi encaminhada em caixas de isopor ao Laboratório de Análises Microbiológicas.

\subsection{Diluições do EM utilizados para biorremediação}

As concentrações de ME utilizadas para biorremediar a água da Barragem (BA) foram: 99,7\% de BA + 0,3\% de EM; 99\% de BA + $1 \%$ de EM; $97 \%$ de BA + 3\% de EM; $80 \%$ de BA + 20\% de EM; $75 \%$ de BA + 25\% de EM; $70 \%$ de BA + $30 \%$ de $\mathrm{EM} ; 65 \%$ de $\mathrm{BA}+35 \%$ de EM; $60 \%$ de $\mathrm{BA}+40 \%$ de EM; $55 \%$ de $\mathrm{BA}+45 \%$ de EM; $50 \%$ de BA + 50\% de EM.

\subsection{Análise microbiológica}

A metodologia utilizada foi a da American Public Health Association (APHA), foram quantificados os coliformes totais e termotolerantes por meio da técnica do número mais provável (NMP) da água da barragem, do EM e das diluições do EM. Após as análises dos testes presuntivos, realizados em diluições seriadas de $10^{-1}, 10^{-2}$ e $10^{-3}$ com Caldo LST (Lauril Triptose) dos tubos com resultado positivo, realizou-se uma alçada em tubos com $10 \mathrm{ml}$ de Caldo Bile 2\% Verde Brilhante (para quantificar coliformes totais) que foram incubados em estufa bacteriológica a $35.5^{\circ} \mathrm{C}$ por até 48 horas; e uma alçada em tubos com $10 \mathrm{ml}$ de caldo EC (Escherichia coli) (para coliformes termotolerantes) os quais foram colocados em banho-maria a $45.5^{\circ} \mathrm{C}$ por até 48 horas.

Os resultados positivos dos testes avaliados foram observados pela formação de bolhas nos tubos de Durham, através da fermentação dos caldos. Os resultados foram comparados com os estabelecidos pela Portaria Brasileira (Portaria de Consolidação) nº 5 do Ministério da Saúde e com a resolução nº 357, 2005.

\subsection{Análise físico-química}

A metodologia utilizada para análise pelo laboratório Central Analítica foi o Standard Methods for the Examination of 
water and Wastewater. Foram avaliados a água da barragem e o EM com os seguintes parâmetros para água da barragem - pH, sólidos totais, sódio, nitrito, nitrato, cloretos, dureza total, cor aparente, condutividade e turbidez.

\section{Resultados e Discussão}

\subsection{Análise microbiológica da água da barragem e do ME}

Verifica-se na tabela 1 que a água da barragem da bananeira possui grupo de coliformes no valor de NMP >1100/mL mostrando 99\% de índice de contaminação. Esse resultado diferiu de Dornellas e Xavier (2008), que avaliando 6 pontos de coleta do riacho Piauí, um deles sendo a água da barragem, verificaram 46,0 NMP/100mL. A barragem foi construída há 19 anos com a finalidade de geração de empregos, renda e lazer, mas, observando os resultados e de acordo com a resolução $\mathrm{n}^{\circ}$ 357 do CONAMA, não se enquadra na classe 1 de águas doces que pode ser destinada ao abastecimento para consumo humano; à recreação de contato primário, tais como natação, esqui aquático e mergulho.

Tabela 1. Avaliação microbiológica e pH da água da barragem e dos microrganismos eficientes, em julho de 2019.

\begin{tabular}{llll}
\hline & NMP/100mL & pH & 7,44 \\
BA & $>1100$ & 3,21 & \\
EM & $<3,0$ & & \\
\hline
\end{tabular}

Fonte: Autores.

O NMP observado nos microrganismos eficientes foi de $<3,0 / 100 \mathrm{~mL}$, comprovando que este se encontra livre do grupo de coliformes. A Escherichia coli é utilizada em todo o mundo como bioindicadora de contaminação fecal, neste sentido ela é um parâmetro biológico que serve para monitorar alimentos, água, biofertilizantes, etc. Os EM são bastante utilizados em práticas agrícolas e na saúde humana, poucas pesquisas relatam análises microbiológicas. O estudo mostra que ele pode ser recomendado para todos os fins como condicionador de solos, descontaminação da água e como biofertilizante de plantas.

Darolt e Neto (2006), analisando biofertilizantes anaeróbicos e aeróbicos também verificaram a ausência do grupo coliformes fecais. O processo de fermentação que ocorre para obtenção do biofertilizante pode ser considerado como um dos principais fatores que controlam a sobrevivência de microrganismos patogênicos, o que pode estar relacionado à ação da temperatura que, possivelmente, tenha sido um dos fatores determinantes na destruição de coliformes fecais. Conforme Gotaas (1956) e Kiehl (1985), esses organismos não sobrevivem à temperatura de $65^{\circ} \mathrm{C}$, quando expostos durante 60 minutos. Outra possibilidade seria as interações sinérgicas ou antagônicas entre os microrganismos (Loures, 1988).

Para a utilização de EM nas concentrações de 0,3; 1 e 3\% os valores foram >1100 NMP/100ml, mostrando que a água não foi remediada, ou seja, o auxílio dos microrganismos nessas porcentagens não foi eficaz. Nas concentrações de 20; 25; 30; 35; 40; 45 e 50 o resultado de NMP foi: 15; 7.4; 7.2; 15; 3.6;<3.0;<3.0 para coliformes totais, respectivamente. Já para coliformes termotolerantes, seguindo as mesmas concentrações, os valores foram: 27; 7.4; 7.2; $15 ; 3.6 ;<3.0$ e <3.0 (tabela 2), mostrando-se dentro do exigido pelo CONAMA. 
Tabela 2. Número mais provável (NMP/100mL) de coliformes totais e termotolerantes nas concentrações de 0,$3 ; 1 ; 3 ; 20 ; 25$; $30 ; 35 ; 40 ; 45$ e $50 \%$ dos microrganismos eficientes.

\begin{tabular}{lcc}
\hline BA+EM & $\begin{array}{c}\text { NMP/100mL de coliformes } \\
\text { totais }\end{array}$ & $\begin{array}{c}\text { NMP/100mL de coliformes } \\
\text { termotolerantes }\end{array}$ \\
\hline $99,7 \% \mathrm{BA}+0,3 \% \mathrm{EM}$ & $>1100$ & 6.1 \\
$99 \% \mathrm{BA}+1 \% \mathrm{EM}$ & $>1100$ & $<3,0$ \\
$97 \% \mathrm{BA}+3 \% \mathrm{EM}$ & $>1100$ & 11 \\
$80 \% \mathrm{BA}+20 \% \mathrm{EM}$ & 15 & 27 \\
$75 \% \mathrm{BA}+25 \% \mathrm{EM}$ & 7.4 & 7.4 \\
$70 \% \mathrm{BA}+30 \% \mathrm{EM}$ & 7.2 & 7.2 \\
$65 \% \mathrm{BA}+35 \% \mathrm{EM}$ & 15 & 15 \\
$60 \% \mathrm{BA}+40 \% \mathrm{EM}$ & 3.6 & 3.6 \\
$55 \% \mathrm{BA}+45 \% \mathrm{EM}$ & $<3.0$ & $<3.0$ \\
$50 \% \mathrm{BA}+50 \% \mathrm{EM}$ & $<3.0$ & $<3.0$ \\
\hline
\end{tabular}

As diluições seriadas pela técnica de NMP foram realizadas em alíquotas de 0,$1 ; 0,01$ e 0,001. Fonte: Autores.

$\mathrm{Na}$ biorremediação, os organismos vivos são utilizados tecnologicamente para remover e reduzir poluentes ambientais. É uma alternativa ecologicamente mais adequada e eficaz para o tratamento de ambientes contaminados com moléculas orgânicas de difícil degradação, metais pesados, denominados recalcitrantes, que podem ser de origem natural, sintetizadas pelo metabolismo biológico (Santos et al. 2009). Lacerda et al. (2021) mostram que a biorremediação pode ser uma tecnologia utilizada para substituir técnicas empregadas em remediação in situ (como exemplo da barreira reativa) e ex situ (dessorção térmica, incineração e outros) devido a sua capacidade de sustentabilidade.

Os EM em estudo mostraram sua capacidade e eficiência em ser usado como biorremediador. De acordo com Bonfim et al. (2011), a produção de EM pela agricultura familiar é uma tecnologia acessível e de baixo custo. Como já salientado, também foram realizadas análises físico-químicas, tanto da água da barragem, quanto do EM, e apenas baseando-se nos resultados da tabela 3, a água da barragem seria satisfatória para consumo humano, estando dentro do recomendado pela Portaria Brasileira de Consolidação n 5 do Ministério da Saúde, no entanto, o trabalho aqui apresentado é de cunho meramente investigativo e sem objetivo de classificar com precisão a potabilidade da água da barragem da Bananeira, onde também seria necessário mensurar a presença de outros químicos inorgânicos, bem como os metais pesados que podem ser advindos de solo contaminado por pesticidas, da agricultura local. 
Tabela 3. Parâmetros físico-químicos da água contaminada e dos microrganismos eficientes (EM).

\begin{tabular}{lll}
\hline Parâmetros & $\begin{array}{l}\text { Água contaminada } \\
\text { por dejetos animais }\end{array}$ & $\begin{array}{l}\text { Microrganismos } \\
\text { Eficientes (ME) }\end{array}$ \\
\hline pH* & 7,44 & 3,21 \\
Sólidos totais $(\mathrm{mg} / \mathrm{L})$ & 585,2 & 2988,3 \\
Sódio $(\mathrm{mgNa} / \mathrm{L})$ & 108,3 & 118 \\
Nitrito em $\mathrm{N},(\mathrm{mg} / \mathrm{L})$ & 0,06 & 0,32 \\
Nitrato em $\mathrm{N},(\mathrm{mg} / \mathrm{L})$ & 0,54 & 21,28 \\
Cloretos $(\mathrm{mgCl} / \mathrm{L})$ & 185,64 & 1115,84 \\
Dureza total $(\mathrm{mgCaCO} / \mathrm{L})$ & 134,00 & 1400,00 \\
Condutividade $(\mu \mathrm{S} / \mathrm{cm})$ & 780 & 3984 \\
Turbidez $(\mathrm{NTU})$ & 12,99 & 206,00 \\
\hline
\end{tabular}

$\mathrm{pH}^{*}$ - Potencial hidrogeniônico. Fonte: Autores.

Segundo Lenzi et al. (2014), a acidez de águas naturais provém de ácidos fracos, tais como, gás carbônico dissolvido e

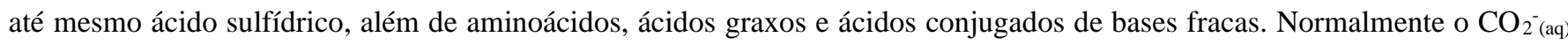
provém da atmosfera ou do próprio corpo d'água causado pela ação de microrganismos aeróbicos, o que justifica a mudança de pH da água da BA quando comparado ao EM.

Atualmente, a água da barragem da Bananeira é utilizada apenas para irrigação, no entanto, pelos resultados microbiológicos, ela não pode ser utilizada para este fim. Na concentração de 100\%, o EM, pelas características físicoquímicas apresentados na tabela 3, não pode ser utilizado para consumo humano e nem para irrigação. De modo geral torna-se complexa fazer uma comparação da água com uma solução de microrganismos eficientes. São necessários estudos de parâmetros físico-químicos em diversas diluições do EM.

\section{Conclusão}

Como resultados preliminares da ação dos microrganismos eficientes, na biorremediação da água, temos que o melhor NMP observado foi de $<3,0 / 100 \mathrm{~mL}$ mostrando que este se encontra livre do grupo coliformes, resultado inicialmente satisfatório, apesar da necessidade de baixas concentrações de diluições, sendo necessário posteriormente estudos de viabilidade econômica $v s$ produção, uma vez que a remediação de água contaminada acontece em concentrações graduais de no mínimo $25 \%$ de fermentado de microrganismos eficientes, que pode remediar até $78 \%$ do grupo coliformes. Os resultados microbiológicos mostraram que a água da barragem da Vila Bananeira possui grupo de coliformes no valor de NMP >1100/mL mostrando $99 \%$ de índice de contaminação, assim, de acordo com a resolução no 357 do CONAMA, não se enquadra na classe 1 de águas doces, tornando seu uso inviável tanto para irrigação quanto para consumo humano.

Conclui-se que, de acordo com os parâmetros físico-químicos avaliados, a água da barragem é satisfatória para consumo humano, estando dentro do recomendado pela Portaria Brasileira (Portaria de Consolidação) n ${ }^{\circ} 5$ do Ministério da Saúde, porém, é necessário análises de outros constituintes químicos para total classificação de potabilidade e mais estudos com as diluições do EM que mostrarão a correlação da formação de ácidos fracos diluídos em consequência da ação dos microrganismos eficientes, além de outros estudos utilizando fertilizantes orgânicos como fonte de biorremediação para águas poluídas. Por último, e não menos importante, aponta-se para necessidade de finalização do projeto inicial da barragem da Vila 
Bananeira, para que esta venha a contribuir com seu papel social e justificar seu investimento de aproximadamente $\mathrm{R} \$ 20$ milhões de reais.

\section{Agradecimentos}

Os autores agradecem ao apoio financeiro da FAPEAL e iniciativa do grupo de pesquisa ECOSISTEM.

\section{Referências}

APHA - American Public Health Association. Eaton, A. D. (2005). Standard methods for the examination of water and wastewater. 21 ${ }^{\text {a }}$ ed. Washington: APHA. 1082 p.

Bonfim, F. P. G., Honório, I. C. G., Reis, I. L., Pereira, A. J., Souza D. B. (2011). Caderno dos microrganismos eficientes (EM): instruções práticas sobre uso ecológico e social do EM. Universidade Federal de Viçosa: Departamento de Fitotecnia.

CONAMA - Conselho Nacional do Meio Ambiente. Resolução n ${ }^{\circ}$ 357, de 17 de março de 2005 - Classificação dos corpos de água e diretrizes ambientais para o seu enquadramento.

Cotta, J. A. de O., Lemos, G. S., \& Lima, E. N. (2020). Ensaios ecotoxicológicos de solo contaminado por diesel submetido à degradação pela espécie Eisenia fétida. Research, Society and Development, 9(2), e106922122. https://doi.org/10.33448/rsd-v9i2.2122

Cuba, R. M. F., Cintra, T. S., Paiva, D. C. C., Téran, F. J. C. (2019). Influência do etanol como cosubstrato na biorremediação de água contaminada com formulação comercial à base de glifosato. Engenharia Sanitária e Ambiental. 5(24). https://doi.org/10.1590/S1413-41522019183574

Darolt, M. R., Neto, E. A. T. (2006). Biofertilizantes: Caracterização química, qualidade sanitária e eficiência em diferentes concentrações na cultura da alface. [Dissertação de Mestrado] (Relatório de Pesquisa) - Universidade Federal do Paraná.

Dornellas, P. C., Xavier, R. A. (2008). Efeito do crescimento urbano na qualidade das águas do riacho Piauí, Arapiraca-AL. Revista de Geografia. Recife: UFPE - DCG/NAPA, 2(25).

Gotaas, H. B. (1956). Composting; sanitary disposal and reclamation of organic wastes. Geneva. 205 p. World Health Organization.

Hunt, M. E., Rice, E. W. (2005). Microbiological examination. In: EATON et al. Standard Methods for the Examination of Water e Wastewater, (21 ${ }^{\circ}$ ed).

Kiehl, E. J. Fertilizantes orgânicos. (1985). São Paulo: Ceres, 492p.

Lacerda, E. M. C., Santana, W. L. A. M. de, Cardoso Filho, M. S., Santos, N. C. P. dos, \& Moreira, Ícaro T. A. (2021). Processos enzimáticos na biorremediação e fitorremediação de petróleo em sedimentos de manguezal: uma revisão. Research, Society and Development, 10(11), e526101119944. https://doi.org/10.33448/rsd-v10i11.19944

Lenzi, E., Favero, L. O. B., Luchese, E. B. (2014). Introdução à Química da Água: Ciência, Vida e Sobrevivência; ISBN 978-85-2161679-5, LTC Livros Técnicos e Científicos Editora Ltda. p. 572 -574.

Loures, E. G. (1988). A microbiota dos solos tropicais: caracterização dos organismos do solo. Agricultura Tropical. ABEAS, 74 p.

Moraes, S. L., Teixeira, C. E., Maximiano, A. M. de S. (2014). (Org.). Guia de elaboração de planos de intervenção para o gerenciamento de áreas contaminadas. São Paulo: IPT, BNDES, 2014.

Moreira, C. A., Dourado, J. C. (2007). Monitoramento da atenuação natural de pluma de contaminação pelo método de radar de penetração no solo (GPR). Revista Brasileira de Geografia. 4(25). São Paulo.

Pereira, L. T. C., Lemos, J. L. S. (2004). Degradação de hidrocarbonetos de petróleo por Aspergillus Niger e Penicillium Corylophilum. In: JORNADA DE INICIAÇÃO CIENTÍFICA, 12., Rio de Janeiro. Anais... Rio de Janeiro: CETEM/MCT.

Santos, R. N., Alves, A. O., Silveira, E. B. (2009). Microrganismos de uso biotecnológico. Anais da IX JEPEX.

Santos, E. H. M., Griebeler, N. P., Oliveira, L. F. C. (2010). Relação entre uso do solo e comportamento hidrológico na Bacia Hidrográfica do Ribeirão João Leite. Revista Brasileira Engenharia Agrícola Ambiental. 8 (14). 826-834.

Silva, R. M. A., Andrade, P. R. G. S. (2014). Simulação da operação de reservatório na bacia do rio Pardo-BA: avaliação da segurança hídrica para o abastecimento público. In: XII Simpósio de Recursos Hídricos do Nordeste. 2014, Natal. Anais, Natal: ABRH. p. 10.

Siqueira, A. P. P., Siqueira, M. F. B. (2013). Bokashi: adubo orgânico fermentado. Niterói: Programa Rio Rural, 16 p, 2013.

Tavares, S. R. L. (2013). Técnicas de remediação. In: Tavares, S. R. L. (Org.). Remediação de solos e águas contaminadas: conceitos básicos e fundamentos. São Paulo: Clube de Autores.

Washington: American Public Health Association (APHA), American Water Works Association (AWWA) e Water Environment Federation (WEF). (2005). Part 9000, p. $9.1-9.169$. 